\title{
Nicorandil Preserves Coronary Collateral Circulation Compared to Nitroglycerin Even in Systemic Hypotension: A Real-Time Myocardial Contrast Echocardiographic Study
}

\author{
Akiko Iwata, Fuminobu Ishikura, Kentaro Ohtani, Tsutomu Toshida, \\ Toshihiko Asanuma and Shintaro Beppu \\ Division of Functional Diagnostic Science, Graduate School of Medicine \\ Osaka University, Suita, Japan
}

\begin{abstract}
Background. Although collateral circulation is important for myocardial salvage in acute coronary syndrome, effects of collaterals are significantly reduced by systemic hypotension. The objective of this study is to evaluate the effects of nitroglycerin and nicorandil on micro-collateral circulation during systemic hypotension.

Methods. Real-time myocardial contrast echocardiography (MCE) was performed in 15 open-chest dogs during occlusion of the left circumflex artery using a snare. Ischemic area was identified using high ultrasound power exposure (burst) just after coronary occlusion. Micro-collateral vessels were recognized by re-opacification during 30 seconds of occlusion. Final subjects for this study were 8 of the 15 dogs that displayed micro-collateral vessels. Flow volume of the donor artery, i.e., the left anterior descending coronary artery (LAD), was measured. Video intensity (VI) were measured and percent wall thickening (\%WT) was calculated. Replenishment curves of VI for the ischemic area after burst were obtained and used in the exponential function $\mathrm{y}=\mathrm{A}\left(1-\mathrm{e}^{-\beta t}\right)$, for estimation of myocardial blood flow volume $(A X$ $\beta$ ). These indices were compared at control and during hypotension after nitroglycerin $(0.01-0.02 \mathrm{mg} / \mathrm{kg})$ or nicorandil (0.1-0.2 $\mathrm{mg} / \mathrm{kg})$ administration.

Results. Equivalent hypotension was induced by each drug. VI, \%WT and $A \times \beta$ at the ischemic area were significantly decreased after nitroglycerin $(p<0.05)$ administration, but not after nicorandil. Nicorandil increased LAD flow from 6.1 $\pm 2.3 \mathrm{ml} / \mathrm{min}$ to $8.8 \pm 2.1 \mathrm{ml} / \mathrm{min}$, but nitroglycerin did not.

Conclusion. Compared with nitroglycerin, nicorandil offers better preservation of coronary collateral circulation, even during systemic hypotension.

(J Echocardiogr 2004; 2: 90-98)
\end{abstract}

Key words: myocardial contrast echocardiography, collateral circulation, nicorandil, nitroglycerin

Two main determinants of both infarction size and amount of viable myocardium following acute coronary occlusion are duration of occlusion and extent of collateral blood flow within the occluded vascular bed $[1,2]$. Extent of collateral flow depends on perfusion pressure, and might deteriorate under systemic hypotension [3], but most therapeutic agents for acute coro-

Received November 11, 2004; revision received November 27, 2004; accepted December 2, 2004

Address for correspondence: Shintaro Beppu, MD

Division of Functional Diagnostic Science,

Graduate School of Medicine, Osaka University,

1-7 Yamadaoka, Suita 565-0871, Japan.

TelePhone: +81-6-6879-2561

Fax: +81-6-6879-2561

E-mail: beppu@sahs.med.osaka-u.ac.jp

(C) 2003 Journal of Echocardiography nary syndrome induce systemic hypotension. Determining the influence of these agents on the micro-collateral circulation under various hemodynamic states is thus extremely important.

Myocardial contrast echocardiography (MCE) offers a suitable imaging technique for quantitative assessment of myocardial perfusion. Wei et al. described a method for measuring flow velocity, myocardial blood volume, and myocardial blood flow (MBF) from a replenishment curve of myocardial video intensity (VI) using MCE [4]. The curve is fitted to the exponential function $\mathrm{y}=\mathrm{A}\left(1-\mathrm{e}^{-\beta t}\right)$, where A is plateau VI (representing myocardial blood volume), and $\beta$ represents flow velocity. The product $A \times \beta$ denotes myocardial blood flow volume $(\mathrm{MBF})$. Analysis of the replenishment curve is thus useful for diagnosing coronary stenosis [4]. 
Real-time MCE, moreover, allows continuous and simultaneous visualization of myocardial perfusion and wall motion, and can provide quantitative analysis of myocardial blood flow from the replenishment curve of VI $[5,6]$. Myocardial perfusion through the collateral circulation can also be observed using MCE [7-17], and real-time MCE may be effective for analyzing collateral perfusion at various levels of blood pressure.

Nitroglycerin has been used for preventing or eliminating myocardial ischemia for over a century. Nicorandil, a hybrid nitrate and adenosine triphosphate (ATP)-sensitive potassium channel opener [18], has also proven beneficial in treating myocardial ischemia and reperfusion [19-22]. Nicorandil is the first anti-anginal agent to have shown positive effects on prognosis in patients with ischemic heart disease [23]. However, the effect of each agent on the micro-collateral circulation during systemic hypotension is unclear. The present study evaluated differences in the effect of collaterals between nicorandil and nitroglycerin using real-time MCE.

\section{Methods}

\section{Animal preparation}

Subjects in the present study were 15 healthy dogs with a mean $( \pm \mathrm{SD})$ body weight of $13.2 \pm 2.3 \mathrm{~kg}$ (range, 9.0-16.0 kg). The experimental protocol was approved by the Osaka University Medical School Animal Care and Use Committee and complied with Osaka University Medical School guidelines for the care and use of laboratory animals. Dogs were anesthetized using intravenous pentobarbital sodium, with an initial bolus $(35 \mathrm{mg} / \mathrm{kg}$ ) followed by continuous administration throughout the experiment (6-8 $\mathrm{mg} / \mathrm{kg} / \mathrm{h}$ ). Dogs were intubated, and ventilated using an SN-480-3 Harvard-type respirator (Shinano, Tokyo, Japan) supplementing room air, and electrocardiography was continuously monitored. Saline was intravenously administered at approximately $1 \mathrm{ml} / \mathrm{min}$ during the surgical procedure. Catheters were placed in the right femoral vein for drug administration, and in the right femoral artery for recording arterial pressure. Lateral thoracotomy along the $5^{\text {th }}$ intercostal space was performed and the heart was suspended in a pericardial cradle. The proximal portions of the left anterior descending artery (LAD) and left circumflex artery (LCx) were dissected free from surrounding tissues. A hydro-occluder was placed around the proximal portion of the LCx. Coronary flows through the LAD and
LCx were measured using an ultrasonic transit-time flowmeter (2SB1212 probe \& T106 blood flowmeter; Transonic Systems, NY, USA).

\section{Myocardial contrast echocardiography}

Real-time MCE was performed with a Sequoia 512 ultrasound platform (Siemens) using a coherent contrast imaging method (mechanical index, 0.1; frame rate, $20 \mathrm{~Hz}$ ). The transducer (3v2C probe, 1.75/3.5 $\mathrm{MHz}$ ) was fixed on the surface of the left ventricular anterior wall to record a short-axis view at the mid-papillary muscle of the left ventricle by a clump. Optimal depth, focus point, and gain were set at the beginning of each experiment and kept constant throughout the experiment.

Optison (Molecular Biosystems, San Diego, USA), which consists of albumin-coated microbubbles containing air and perfluorocarbon with a mean size of $3.9 \mu \mathrm{m}$ and a mean concentration of $8 \times 10^{8} / \mathrm{mL}$, was used. A 10-fold dilution of Optison was infused at a constant rate of $60-120 \mathrm{ml} / \mathrm{h}$ from a peripheral vein to obtain adequate real-time MCE imaging. All images were recorded on videotape using an SVO-9600 S-VHS recorder (SONY, Tokyo, Japan).

Video intensity (256 gray scale) of myocardial opacification at end-systole was measured off-line using a color cardiology workstation (Tom Tec Imaging Systems, USA) [24].

\section{Definition of good collateral perfusion}

The ischemic area following coronary occlusion was easily identified as the non-opacified area after complete bubble destruction by transient high ultrasound power exposure (burst; mechanical index, 1.9). Presence of natural collateral flow to the ischemic area was determined by de novo opacification after $30 \mathrm{sec}-$ onds of LCx occlusion. Well-developed collateral perfusion was defined when VI after 30 seconds of LCx occlusion was $>15 \%$ in area of the LAD. No opacification after burst indicated absence of collateral flow (Figure 1). Of the 15 dogs, 8 displayed well-developed collateral perfusion, and these dogs represented the final subjects for this study.

\section{Analysis of MCE}

Areas at risk were divided into 3 regions (core and border zones 1 and 2; Figure. 1) to evaluate zone specificity in the ischemic area. Time-VI plots were obtained in each region, and were fitted to the exponential function $y=A\left(1-e^{-\beta t}\right)$, where $y$ is VI, A is plateau VI (myocardial blood volume), $\beta$ is up-slope (velocity of blood flow) and the product $\mathrm{A} \times \beta$ denotes MCE- 
derived MBF [4].

\section{Percent wall thickening}

Percent wall thickening (\%WT) of ischemic and normal regions was measured 30 seconds after LCx occlu- sion. The \%WT was calculated as: (wall thickness at end-systole - wall thickness at end-diastole) $\times 100 /$ wall thickness at end-diastole. Wall thickness was measured from the outside edge of the epicardial echo to
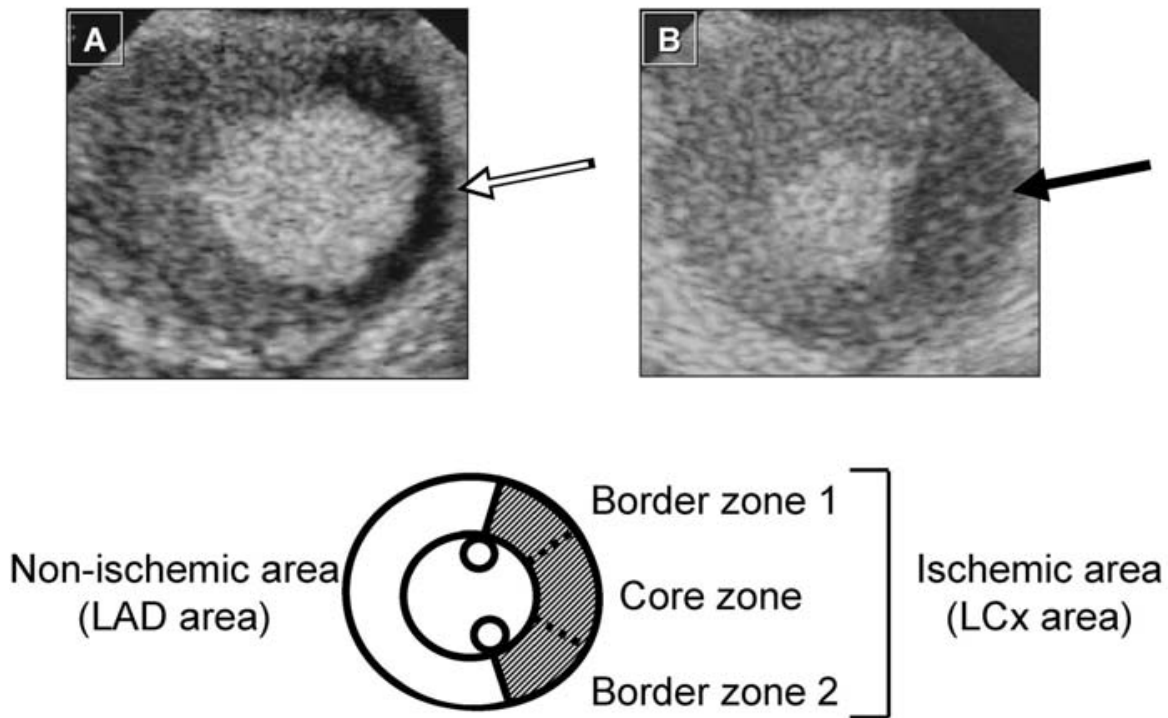

Fig. 1. MCE 30 seconds after LCx occlusion followed by "burst" procedure

A) Poor collateral perfusion. Wall thickness of the area at risk is thin (open arrow).

B) Well-developed collateral perfusion. Wall thickness of the area at risk is not thin (solid arrow). Ischemic area is divided into three zones (core and border zones 1 and 2).

$L A D$, left anterior descending coronary artery; LCx, left circumflex coronary artery

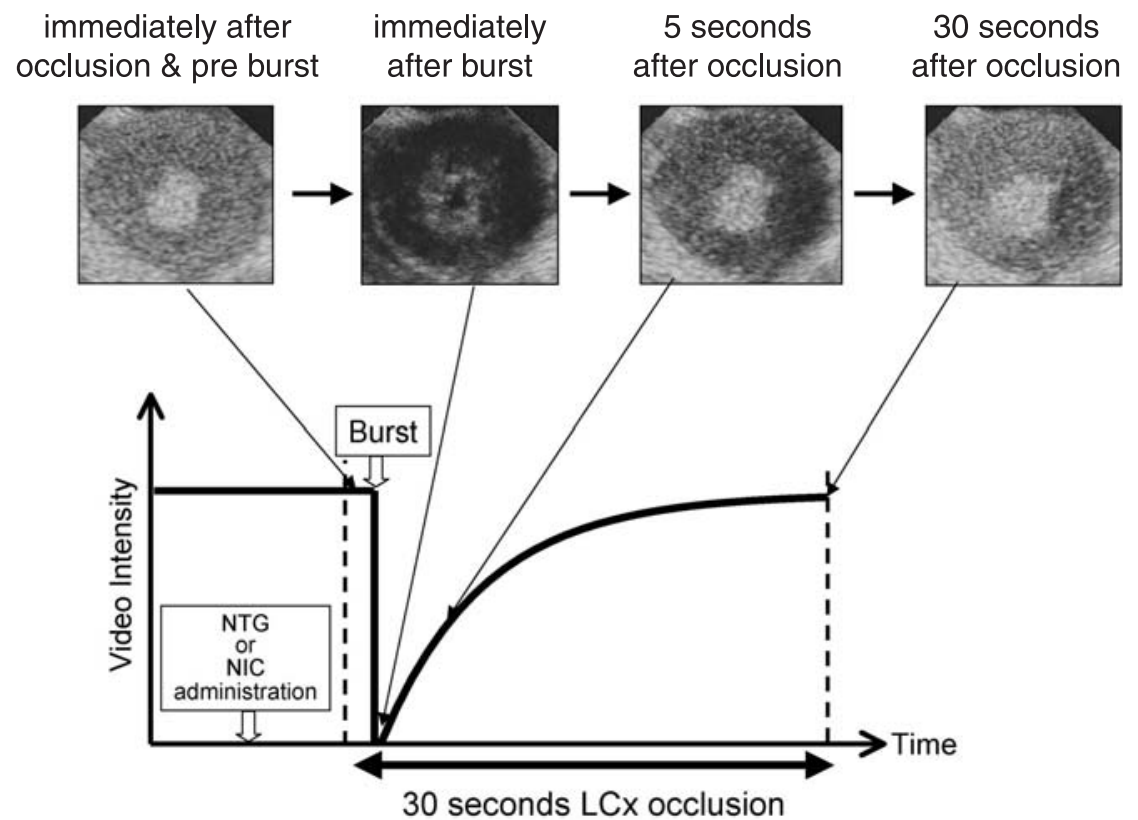

Fig. 2. Protocol design

NTG, nitroglycerin; NIC, nicorandil 
the inside edge of the endocardial echo using a caliper on the two-dimensional echocardiography.

\section{Experimental protocol}

Heart rate, systemic blood pressure, and coronary flow volume for the $\mathrm{LAD}$ and $\mathrm{LCx}$ were continuously monitored throughout the experiment (Figure 2). As a control protocol before drug administration, real-time MCE was performed during 30 seconds after $\mathrm{LCx}$ occlusion. After release of LCx occlusion and complete recovery of hemodynamics, high-dose nitroglycerin $(0.01-0.02 \mathrm{mg} / \mathrm{kg})$ or high-dose nicorandil (0.1-0.2 $\mathrm{mg} / \mathrm{kg}$ ) was administered. Hypotension was defined as mean blood pressure (mBP) at under $50 \mathrm{mmHg}$. After stabilizing hypotension, real-time MCE was performed in a similar method to the control stage. After release of LCx occlusion and complete recovery of hemodynamics for about 60 minutes, the protocol of other drug was performed. Order of drug administration was random.

\section{Statistical methods}

Data are expressed as mean \pm SD. Hemodynamics before and after administration of each drug were compared using the paired Student's t-tests. Values of $\mathrm{p}<0.05$ were considered statistically significant for all comparisons.

\section{Results}

\section{Hemodynamic changes (Table 1)}

No significant differences in heart rate were identified 30 seconds after LCx occlusion in all dogs between the control, nitroglycerin and nicorandil administrations. Both nitroglycerin and nicorandil significantly reduced $\mathrm{mBP}$ in all dogs $(75 \pm 16 \mathrm{mmHg}$ to $41 \pm 8 \mathrm{mmHg}, 72 \pm 13 \mathrm{mmHg}$ to $47 \pm 7 \mathrm{mmHg}$, respectively; $\mathrm{p}<0.01)$. LAD flow after LCx occlusion increased significantly from $6.1 \pm 2.3 \mathrm{ml} / \mathrm{min}$ to $8.8 \pm 2.1 \mathrm{ml} / \mathrm{min}$ after nicorandil administration $(\mathrm{p}<0.05)$, but remained unchanged after nitroglycerin administration.

Table 1. Effects of agents on hemodynamic changes during LCx occlusion

\begin{tabular}{lcccc}
\hline & \multicolumn{2}{c}{ Nitroglycerin $(\mathrm{n}=8)$} & \multicolumn{2}{c}{ Nicorandil $(\mathrm{n}=8)$} \\
\hline & Before & After & Before & After \\
Heart rate $(\mathrm{bpm})$ & $105 \pm 13$ & $104 \pm 12$ & $103 \pm 12$ & $102 \pm 12$ \\
SBP (mmHg) & $100 \pm 18$ & $63 \pm 11^{\dagger}$ & $98 \pm 21$ & $68 \pm 13^{*}$ \\
DBP (mmHg) & $62 \pm 16$ & $30 \pm 7^{*}$ & $60 \pm 13$ & $36 \pm 6^{*}$ \\
Mean BP (mmHg) & $75 \pm 16$ & $41 \pm 8^{*}$ & $72 \pm 13$ & $47 \pm 7^{*}$ \\
LAD flow (ml/min) & $6.0 \pm 1.9$ & $5.3 \pm 1.3$ & $6.1 \pm 2.3$ & $8.8 \pm 2.1^{\dagger}$ \\
\hline
\end{tabular}

* Significantly different $(\mathrm{p}<0.01)$ from before administration

$\dagger$ Significantly different $(\mathrm{p}<0.05)$ from before administration

Table 2. Analysis of video intensity at non-ischemic and ischemic area

\begin{tabular}{cllll}
\hline & \multicolumn{2}{c}{ Nitroglycerin $(\mathrm{n}=8)$} & \multicolumn{2}{c}{ Nicorandil $(\mathrm{n}=8)$} \\
\hline & Before & After & Before & After \\
Non-ischemic area & $72 \pm 15$ & $75 \pm 16$ & $69 \pm 17$ & $80 \pm 10^{\dagger}$ \\
Ischemic area & & & & \\
Border zone 1 & $34 \pm 15^{* \ddagger}$ & $24 \pm 10^{* \ddagger \#}$ & $36 \pm 16^{* \ddagger}$ & $38 \pm 13^{* \S}$ \\
Core zone & $24 \pm 14^{*}$ & $17 \pm 12^{* \#}$ & $25 \pm 16^{*}$ & $27 \pm 14^{*}$ \\
Border zone 2 & $39 \pm 17^{* \ddagger}$ & $26 \pm 14^{* \S}$ & $34 \pm 15^{* \S}$ & $40 \pm 13^{* \ddagger}$ \\
\hline
\end{tabular}

$* \mathrm{p}<0.01$ vs. non-ischemic area

$\S p<0.05$ vs. core zone in each of the protocols

$\ddagger \mathrm{p}<0.01$ vs. core zone in each of the protocols

\# $\mathrm{p}<0.05$ vs. before drug administration

$\dagger^{\dagger}<0.01$ vs. before drug administration 


\section{Real-time MCE (Table 2)}

Figure 3 shows perfusion in ischemic and nonischemic areas at the control stage, and during systemic hypotension following nitroglycerin and nicorandil administration. Before burst, no ischemic areas were recognized even after complete occlusion of LCx. All bubbles in the myocardium were destroyed by burst. The ischemic area displayed gradual re-opacification, indicating the presence of collateral circulation. In the representative case, intensity at the ischemic area 30 seconds after burst was low during hypotension caused by nitroglycerin, but it was almost the same in the control state and hypotension caused by nicorandil. VI values in non-ischemic areas and 3 zones of ischemic areas 30 seconds after LCx occlusion are summarized in Table 2. VI for each zone of the ischemic area was significantly lower than VI at the non-ischemic area, irrespective of drug administration. In the ischemic area, VI was significantly lower in the core zone than in either border zone. After nitroglyc-

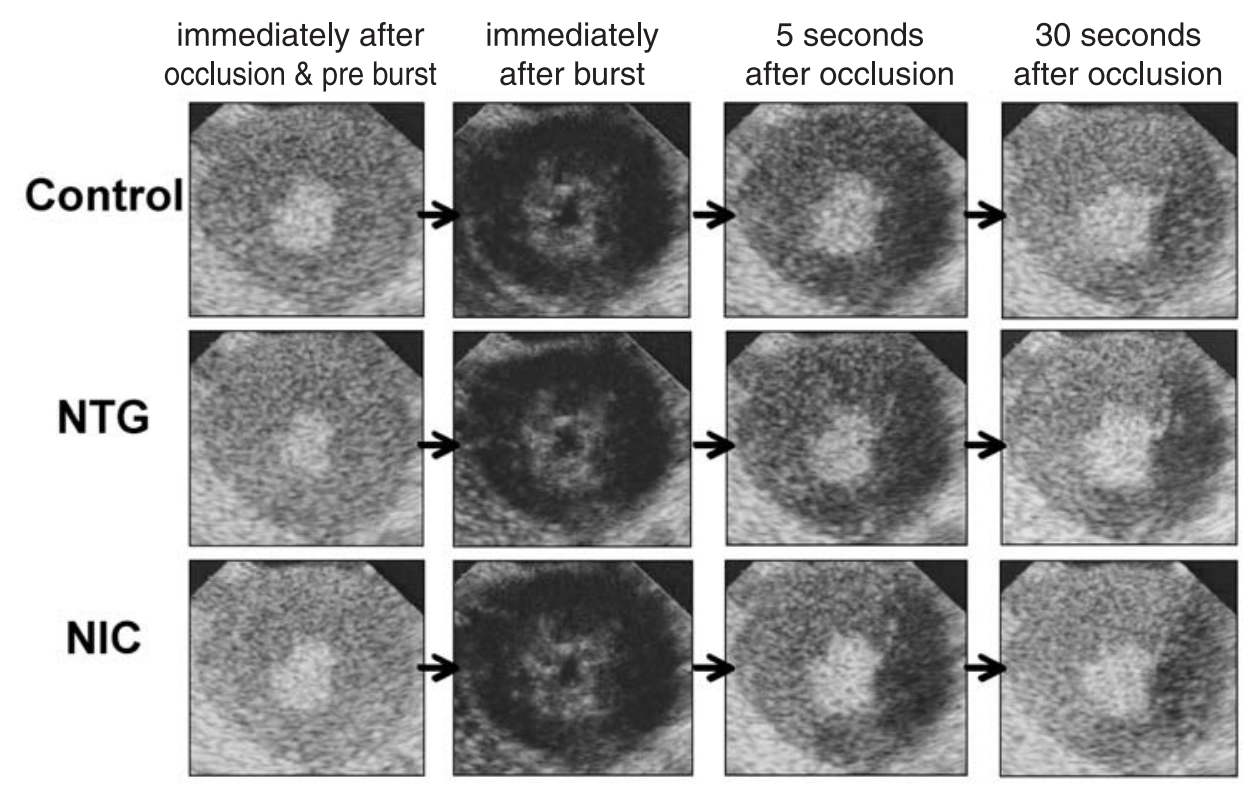

Fig. 3. Temporal changes in opacification at control stage and after administration of NTG and NIC

While opacification of the area at risk is weaker after NTG administration than at the control stage, opacification after NIC administration is almost the same as at the control stage, despite hypotension.

Table 3. Analysis of \% wall thickening at non-ischemic and ischemic area

\begin{tabular}{ccccc}
\hline & \multicolumn{2}{c}{ Nitroglycerin $(\mathrm{n}=8)$} & \multicolumn{2}{c}{ Nicorandil $(\mathrm{n}=8)$} \\
\hline & Before & After & Before & After \\
Non-ischemic area & $59 \pm 12$ & $57 \pm 9$ & $74 \pm 31$ & $70 \pm 19$ \\
Ischemic area & & & & \\
Border zone 1 & $33 \pm 10^{\S \#}$ & $24 \pm 5^{\S \dagger \dagger}$ & $35 \pm 9^{* \dagger}$ & $37 \pm 9^{\S \#}$ \\
Core zone & $23 \pm 8^{\S}$ & $17 \pm 11^{\S \dagger}$ & $26 \pm 8^{\S}$ & $25 \pm 9^{\S}$ \\
Border zone 2 & $32 \pm 9^{\S \ddagger}$ & $24 \pm 8^{\S \dagger}$ & $33 \pm 12^{\S \ddagger}$ & $38 \pm 7^{\S \#}$ \\
\hline
\end{tabular}

\footnotetext{
$* \mathrm{p}<0.05$ vs. non-ischemic area

$\S \mathrm{p}<0.01$ vs. non-ischemic area

$\ddagger \mathrm{p}<0.05$ vs. core zone in each of the protocols

$\# p<0.01$ vs. core zone in each of the protocols

$\dagger \mathrm{p}<0.05$ vs. before drug administration
} 


\section{MCE parameters at ischemic area}
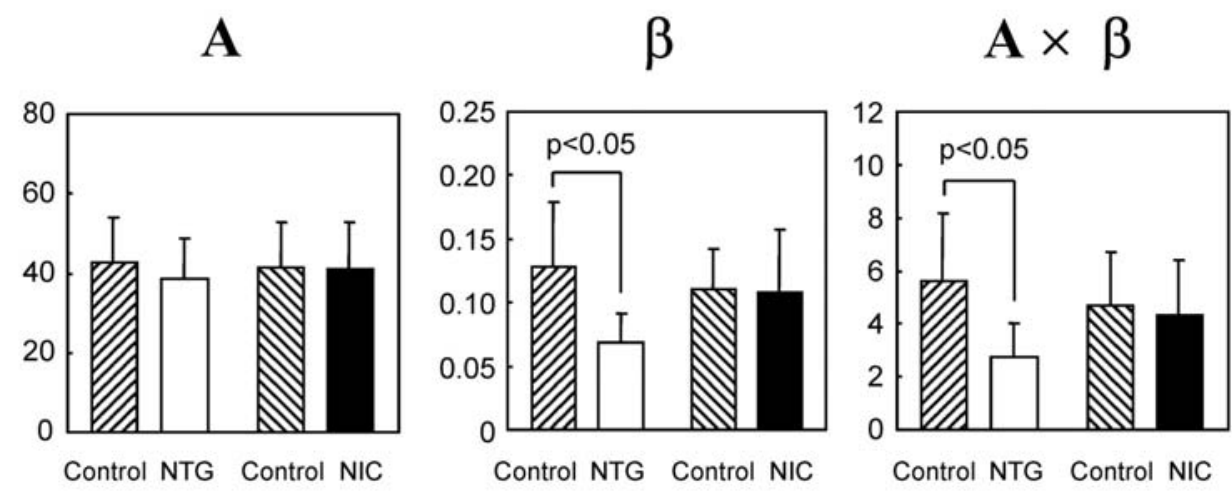

Fig. 4. MCE indices in ischemic area at control stage and during hypotension after administration of NTG and NIC

The $\beta$ and $A \times \beta$ values decreased significantly after NTG administration $(p<0.05)$. However, $A, \beta$ and $A \times \beta$ values after NIC administration were not significantly different from the control stage.

erin administration, $\mathrm{VI}$ in the ischemic area was significantly decreased, while VI in the non-ischemic area was unchanged. After nicorandil administration, however, VI was not decreased at any zone within the ischemic area.

\section{Percent wall thickening (Table 3)}

The \%WT of the ischemic area was significantly lower than that of the non-ischemic area. Moreover, $\% \mathrm{WT}$ of the core zone was the lowest of the ischemic areas. After nitroglycerin administration, \%WT of the ischemic area decreased significantly. After nicorandil administration, however, \%WT was unchanged in all zones of the ischemic area.

\section{MCE parameters from replenishment curve}

Quantitative analysis of the replenishment curve examining A and $\beta$ values were performed only in 7 of 24 regions on the nitroglycerin protocol and 11 of 24 regions on the nicorandil protocol, as not all VI plots could be fitted to the exponential function $\mathrm{y}=\mathrm{A}\left(1-\mathrm{e}^{-\beta t}\right)$, due to extremely low VI.

Mean values of $A, \beta$ and $A \times \beta$ in the ischemic area before and after drug administration are shown in Figure 4. After nitroglycerin administration, $\beta$ and $A \times \beta$ values were significantly decreased $(\mathrm{p}<0.05)$, whereas A did not significantly change. After nicorandil administration, however, neither $\mathrm{A}, \beta$ nor $\mathrm{A} \times \beta$ was changed in the ischemic area.

\section{Discussion}

Collateral circulation through microvessels is crucial for salvaging at-risk myocardium during coronary obstruction. Coronary angiography has been used to examine the presence of collateral vessels. However, numerous papers have declared that $\mathrm{MCE}$ is superior to coronary angiography for examining the significance of collateral perfusion [7-10]. Clinically, both the presence and function of a collateral vessel, such as stability during stress or hypotension, are important. The present study also revealed that real-time MCE can demonstrate recruitment of micro-collateral circulation after coronary occlusion. Viewing real-time images of wall motion and myocardial perfusion has clarified that hypotension reduces micro-collateral perfusion. In this regard, therapeutic agents may exert adverse effects on collateral perfusion, due to vasodilatory effects that induce hypotension.

Video intensity 30 sec. after burst vs. replenishment curve

The product $A \times \beta$ from the replenishment curve is considered to indicate MCE-derived $\mathrm{MBF}$, as many studies have reported that MCE-derived MBF correlates well with radiolabeled microsphere-derived MBF [4, 14-17]. However, Coggins et al. mentioned that the flow speed through the micro-collateral vessels can be extremely slow [14]. VI after coronary 
occlusion did not completely replenish to plateau levels within 30 seconds in 3 dogs in this study. This was the reason for the small number of assessments using the replenishment curve. Nevertheless, the fact that the VI of the plateau level calculated from the equation of the replenishment curve (i.e. A value) did not differ between the 3 conditions in the at-risk area is noteworthy. Only flow speed (i.e., $\beta$ value) slowed during hypotension from nitroglycerin. VI at a certain time after burst corresponds to flow speed when speed is quite slow. VI was measured at 30 seconds after burst in this study, and differences in VI at 30 seconds after burst should indicate differences in flow speed in this study.

\section{Effects of nitroglycerin}

Nitroglycerin acts to relax vascular smooth muscle by increasing cellular cyclic guanosine monophosphate (cGMP), due to stimulation of guanylate cyclase [25]. Nitroglycerin dilates the larger coronary arteries, but exerts only slight effects on precapillary arterioles. Low doses of nitroglycerin therapy during acute myocardial infarction has been shown to decrease infarction size and increase collateral blood flow [25]. However, beneficial effects on infarct size and collateral blood flow are abolished when hypotension is induced by excessive doses of nitroglycerin $[25,26]$. A paradoxical J-curve effect on infarct size in acute myocardial infarction is revealed with progression of nitroglycerin-induced reductions in blood pressure. While a mild reduction in arterial pressure decreases infarct size, a significant drop down to $40 \%$ results in myocardial underperfusion and increases infarct size [25]. These high doses of nitroglycerin produce excessive vasodilatation and loss of coronary autoregulation, so myocardial perfusion becomes dependent on driving perfusion pressure [26]. The present results indicate that \%WT decreases in the ischemic area due to decreased blood volume, flow speed and flow volume, causing a loss of coronary autoregulation during systemic hypotension induced by high-dose nitroglycerin administration.

\section{Effects of nicorandil}

Nicorandil is a hybrid drug combining a nitrate and an ATP-sensitive potassium channel opener [18], and exerts two actions. Vascular smooth muscle is relaxed by increasing cellular cGMP due to stimulation of guanylate cyclase and by hyperpolarization from ATP-dependent opening of $\mathrm{K}^{+}$channels [27]. Hyperpolarization in vascular smooth muscle inhibits
$\mathrm{Ca}^{2+}$ influx via voltage-dependent $\mathrm{Ca}^{2+}$ channels and leads to vasodilatation. Nicorandil can thus dilate both large coronary arteries and small resistance arterioles. Low doses of nicorandil predominantly dilate small arterioles by the ATP-dependent opening of $\mathrm{K}^{+}$channels, whereas high doses of nicorandil dilate the pericardial coronary arteries due to effects on cGMP [27]. Nicorandil has beneficial effects for myocardial ischemia and reperfusion [19-22], including dilation of normal and stenotic segments of coronary arteries [28] and the absence of adverse effects such as coronary steal phenomenon [29]. However, the effects of nicorandil under conditions of systemic hypotension have not been examined previously.

Using a radioactive microsphere technique, Lamping et al. reported that nicorandil increased collateral flow when aortic pressure was maintained at predrug levels, but had no beneficial effects on collateral blood flow during mild hypotension [30, 31]. Conversely, Shiraki et al. reported that intravenous nicorandil decreased peripheral vascular resistance and increased peripheral coronary blood flow, even when systemic pressure fell down [32]. While those studies were limited to normal hearts, not ischemic hearts, our study was applied to the ischemic heart under systemic hypotension. Nicorandil increased blood volume in the donor artery and preserved blood volume, flow speed and flow volume at the ischemic area even during systemic hypotension in this study. Komaru et al. suggested that ATP-sensitive $\mathrm{K}^{+}$channels play an important role in determining coronary microvascular responses to reductions in coronary perfusion pressure [33]. Lamping et al. reported that collateral vessels less than $100 \mu \mathrm{m}$ in diameter dilated under conditions of systemic normotension, and moreover, activation of ATP-sensitive $\mathrm{K}^{+}$channels involves progressive dilation of collateral vessels [34]. Our results indicate that nicorandil preserves the collateral circulation, because the hyperpolarizing effect of nicorandil might dilate small arterioles including collateral vessels without loss of coronary autoregulation even during systemic hypotension.

\section{Limitations of the present study}

The collateral perfusion examined in this study occurred via the existing collateral vasculature. In clinical cases, however, sustained myocardial ischemia caused by severe coronary stenosis could promote the development of new collateral vessels. Collateral flow through the epicardial arteries may thus differ from 
that through intramural vessels. Epicardial collateral flow occurs in the majority of clinical cases, but was not examined in this study.

Myocardial ischemia due to coronary obstruction usually lasts for a number of hours in most clinical cases. We did not examine data over 30 seconds of coronary occlusion, because prolonged ischemia might induce hemodynamic changes or fatal arrhythmia, and examination of drug effects on collateral flow might thus be difficult. A period of 30 seconds might be somewhat brief for evaluating coronary collateral flow dynamics.

This study was applied in ischemic hearts at excessive hypotension. We focused on relationships between drug-induced hypotension and effects on the collateral circulation, without regard to adequate therapeutic dose. Therapeutic effect and hemodynamics should vary according to the dose of drugs administered. Further studies are needed to examine the effects on collateral perfusion with low-dose nitroglycerin or nicorandil at lesser degrees of hypotension.

\section{Conclusion}

This study elucidated differences between the effects of nitroglycerin and nicorandil on micro-collateral circulation during systemic hypotension using real-time MCE. MCE revealed that blood pressure influences coronary collateral circulation and that effects on the collateral circulation was quite different between the two therapeutic agents, nitroglycerin and nicorandil, even when the level of systemic hypotension was equivalent. Compared to nitroglycerin, nicorandil provides better preservation of coronary collateral circulation, even under systemic hypotension.

\section{References}

1. Shaper W, Frenzel H, Hort W. Experimental coronary occlusion, I. Measurement of infarct size. Basic Res Cardiol 1979; 74: 46-53.

2. Reimer KA, Jennings RB. The "wavefront phenomenon" of myocardial ischemic cell death, II. Transmural progression of necrosis within the framework of ischemic bed size (myocardium at risk) and collateral flow. Lab Invest 1979; 40: 633-44.

3. Scheel KW, Mass H, Williams SE. Pressure-flow characteristics of coronary collaterals in dogs. Am J Physiol 1989; 256: H441-H445.

4. Wei K, Jayaweera AR, Firoozan S, Linka A, Skyba DM, Kaul S. Quantification of myocardial blood flow with ultrasound-induced destruction of microbubbles administered as a constant venous infusion. Circulation 1998; 97: 47383.

5. Masugata H, Peters B, Lafitte S, Strachan M, Ohmori K, DeMaria AN. Quantitative assessment of myocardial perfusion during graded coronary stenosis by real-time myocardial contrast echo refillng curves. J Am Coll Cardiol 2001; 37: 262-9.

6. Lafitte S, Masugata H, Peters B, Togni M, Strachan M, Kwan OL, et al. Comparative value of dobutamine and adenosine stress in the detection of coronary stenosis with myocardial contrast echocardiography. Circulation 2001; 103: 2724-30.

7. Lim YJ, Nanto S, Masuyama T, Kodama K, Kohama A, Kitabatake A, et al. Coronary collateral assessed with myocardial contrast echocardiography in healed myocardial infarction. Am J Cardiol 1990; 66: 556-61.

8. Sakata Y, Kodama K, Adachi T, Lim YJ, Ishikura F, Fuji $\mathrm{H}$, et al. Comparison of myocardial contrast echocardiography and coronary angiography for assessing the acute protective effects of collateral recruitment during occlusion of the left anterior descending coronary artery at the time of elective angioplasty. Am J Cardiol 1997; 79: 132933.

9. Sabia PJ, Powers ER, Jayaweera AR, Ragosta M, Kaul S. Functional significance of collateral blood flow in patients with recent acute myocardial infarction. Circulation 1992; 85: 2080-9.

10. Sabia PJ, Powers ER, Ragosta M, Sarembock IJ, Burwell LR, Kaul S. An Association between collateral blood flow and myocardial viability in patients with recent myocardial infarction. N Engl J Med 1992; 327: 1825-31.

11. Kemper AJ, Force T, Kloner R, Gilfoil M, Perkin L, Hale $\mathrm{S}$, et al. Contrast echocardiographic estimation of regional myocardial blood flow after acute coronary occlusion. Circulation 1985; 72: 1115-24.

12. Kaul S, Pandian NG, Guerrero JL, Gillam LD, Okada RD, Weyman AE. Effects of selectively altering collateral driving pressure on regional perfusion and function in the occluded coronary bed in the dog. Circ Res 1987; 61: 7785.

13. Cheirif J, Narkiewicz-Jodko JB, Hawkins HK, Bravenec JS, Quinones MA, Mickelson JK. Myocardial contrast echocardiography: Relation of collateral perfusion to extent of injury and severity of contractile dysfunction in a canine model of coronary thrombosis and reperfusion. J Am Coll Cardiol 1995; 26: 537-46.

14. Coggins MP, Sklenar J, Le E, Wei K, Lindne JR, Kaul S. Noninvasive prediction of ultimate infarct size at the time of acute coronary occlusion based on the extent and magnitude of collateral-derived myocardial blood flow. Circulation 2001; 104: 2471-7.

15. Mills JD, Fisher D, Villanueva FS. Coronary collateral 
development during chronic ischemia: Serial assessment using harmonic myocardial contrast echocardiography. J Am Coll Cardiol 2000; 36: 618-24.

16. Grill HP, Brinkner JA, Taube JC, Walford GD, Midei MG, Flaherty JT, et al. Contrast echocardiographic mapping of collateralized myocardium in humans before and after coronary angioplasty. J Am Coll Cardiol 1990; 16: 1594600.

17. Ha JW, Cho SY, Chung N, Choi DH, Choi BJ, Jang Y, et al. Fate of collateral circulation after successful coronary angioplasty of total occlusion assessed by coronary angiography and myocardial contrast echocardiography. J Am Soc Echocardiogr 2002; 15: 389-95.

18. Taira N. Nicorandil as a hybrid between nitrates and potassium channel activators. Am J Cardiol 1989; 63: 18J24J.

19. Gross GJ, Pieper G, Warltier DC. Comparative effects of nicorandil, nitroglycerin, nicotinic acid, and SG-86 on the metabolic status and functional recovery of the ischemicreperfused myocardium. J Cardiovasc Pharmacol 1987; 10 (Supple. 8): S76-S84.

20. Gross GJ, Warltier DC, Hardman HF. Comparative effects of nicorandil, a nicotinamide nitrate derivative, and nifedipine on myocardial reperfusion injury in dogs. J Cardiovasc Pharmacol 1987; 10: 535-42.

21. Auchampach JA, Cavero I, Gross GJ. Nicorandil attenuates myocardial dysfunction associated with transient ischemia by opening ATP-dependent potassium channels. J Cardiovasc Pharmacol 1992; 20: 765-71.

22. Ito H, Taniyama Y, Iwakura K, Nishikawa N, Masuyama $\mathrm{T}$, Kazuya $\mathrm{T}$, et al. Intravenous nicorandil can preserve microvascular integrity and myocardial viability in patients with reperfused anterior wall myocardial infarction. J Am Coll Cardiol 1999; 33: 654-60.

23. The IONA study group. Effect of nicorandil on coronary artery in patients with stable angina: the Impact Of Nicorandil in Angina (IONA) ramdamized trial. Lancet 2002; 359: 1269-75.

24. Shishido T, Beppu S, Matsuda H, Yutani C, Miyatake K. Extension of hemorrhage after reperfusion of occluded coronary artery: Contrast echocardiographic assessment in dogs. J Am Coll Cardiol 1997; 30: 585-91.

25. Jugdutt BI. Intravenous nitroglycerin unloading in acute myocardial infarction. Am J Cardiol 1991; 68: 52D-63D.

26. Jugdutt BI. Myocardial salvage by intravenous nitroglycerin in conscious dogs: loss of beneficial effect with marked nitroglycerin-induced hypotension. Circulation 1983; 68: 673-84.

27. Holzmann S, Kukovets WR, Braida C, Pöch G. Pharmacological interaction experiments differentiate between glibenclamide-sensitive $\mathrm{K}^{+}$channels and cyclic GMP as components of vasodilation by nicorandil. Eur J Pharmacol 1992; 215: 1-7.

28. Suryapranata H. Coronary haemodynamics and vasodilatory profile of a potassium channel opener in patients with coronary artery disease. Eur Heart J 1993; 14 (suppl B): $16-21$.

29. Krumenacker M, Roland E. Clinical profile of nicorandil: an overview of its hemodynamic properties and therapeutic efficacy. J Cardiovasc Pharmacol 1992; 20 (suppl 3): S93-S102.

30. Lamping KA, Gross GJ. Comparative effects of a new nicotinamide nitrate derivative, nicorandil (SG 75), with nifedipine and nitroglycerin on true collateral blood flow following an acute coronary occlusion in dogs. J Cardiovasc Pharmacol 1984; 6: 601-8.

31. Lamping KA, Warltier DC, Hardman HF, Gross GJ. Effects of nicorandil, a new antianginal agent, and nifedipine a collateral blood flow in a chronic coronary occlusion model. J Pharmacol Exp There 1984; 229: 359-63.

32. Shiraki Y, Akima M, Nabata H, Ohba Y, Hoshino E, Sakai $\mathrm{K}$. The hypotensive mechanisms of the new anti-anginal drug, N-(2-hydroxyethl) nicotinamide nitrate (SG-75) in beagle dogs. Japan. J. Pharmacol 1981; 31: 921-9.

33. Komaru T, Lamping KG, Eastham CL, Dellsperger KC. Role of ATP-sensitive potassium channels in coronary microvascular autoregulatory responses. Circ Res 1991; 69: 1146-51.

34. Lamping KG, Bloom EN, Harrison DG. Regulation of native collateral vessel dilation after coronary occlusion in the dog. Am J Physiol 1994; 266: H769-H778. 